\title{
Awareness of prenatal genetic tests and attitude towards termination of pregnancy regarding fetal congenital heart defects among clinicians in China: a multi-center cross-sectional study
}

jian wang

Shanghai Jiaotong University School of Medicine Xinhua Hospital zhuoyan li

Shanghai Jiaotong University School of Medicine Xinhua Hospital jianping yang

Shanghai Jiaotong University School of Medicine Xinhua Hospital yanan lu

Shanghai Jiaotong University School of Medicine Xinhua Hospital yurong wu

Shanghai Jiaotong University School of Medicine Xinhua Hospital

ailan xie

Wenzhou Medical University

yongwei zhang

Children's Hospital of Shanghai

jiannong chai

changshu hospital

bo han

shandong province hospital

yuqing zhou

shanghai changning maternal hospital

tiangang li

Lanzhou Branch National Science Library Chinese Academy of Sciences

sun chen

Shanghai Jiaotong University School of Medicine Xinhua Hospital

kun sun ( $\nabla$ sunkun@xinhuamed.com.cn )

Shanghai Jiaotong University School of Medicine Xinhua Hospital

Research article 
Keywords: congenital heart disease, prenatal genetic test, prenatal genetic counseling, termination of pregnancy

Posted Date: March 3rd, 2020

DOI: https://doi.org/10.21203/rs.3.rs-15798/v1

License: (a) (i) This work is licensed under a Creative Commons Attribution 4.0 International License. Read Full License 


\section{Abstract}

Background: This study was aim to figure out the level of awareness about providing genetic tests and the attitude towards termination of pregnancy (TOP) regarding fetal congenital heart defects (CHD) among clinicians in China.

Methods: A total of 501 clinicians were divided into 2 groups: clinicians whose working hospital were available of prenatal genetic service were assigned into group 1, whose working hospital were not available of prenatal genetic service were assigned to group 2. The level of awareness and the attitude were evaluated according to scores of a questionnaire.

Results: Among the 501 total clinicians, only $27.94 \%$ received a high score and $53.09 \%$ received a medium score, indicating a low level of awareness about prenatal genetic tests among clinicians. $31.62 \%$ clinicians in group 1 , but only $24.19 \%$ in group 2 showed good awareness of providing genetic tests $(P=0.023)$. About $44.3 \%$ of clinicians in group 1, but only by $29.4 \%$ of those in group 2 held optimistic attitudes towards minor CHDs and were less favor of TOP in minor CHDs $(P=0.023)$.

Conclusion: Prenatal genetic tests and counseling in hospital are necessary and should be improved in the management of fetal CHD in China.

\section{Introduction}

Congenital heart disease (CHD) is one of the most common birth defects and heritable genetic factors play an important role in its etiology[1]. chromosomal abnormalities are present in 15-30\% of fetuses with isolated CHD. The most common chromosomal abnormalities accompanying CHDs are trisomies 21, 18, and 13, monosomy $X$, and 22q11.2 deletion[2]. In the presence of chromosomal abnormalities, fetuses face unfavorable outcomes.

Prenatal detection of CHD allows for prompt medical intervention after birth, and prenatal genetic tests help to reveal the genetic origin of a huge number of $\mathrm{CHD}$ conditions. During clinical practice, healthcare professionals should inform pregnant women about the availability of genetic tests [4] and once an accurate diagnosis of prenatal $\mathrm{CHD}$ is made, the condition should be communicated to the family via prenatal counseling. It was reported that prenatal genetic tests and counseling should be recommended for all fetal CHD [3]. However, no official statistics are available on the number of healthcare professionals (e.g., physicians, nurses, laboratory technicians) providing genetic counseling services in China [5]. Moreover, unlike in the United States of America and Europe, there are no specialty-trained geneticists or genetic counselors in China, and genetic counseling is primarily provided by pediatricians or obstetricians. Meanwhile, though there are several effective and universal ways of genetic tests available in hospitals, numerous challenges and barriers have been encountered in transforming genetic test information to medical practice. Thus, the genetic service (access to genetic tests and counseling) system has not been well established yet in China. 
Effective prenatal counseling is one most important roles of clinicians during clinical practice [6]. In a consensus on diagnosis and perinatal management of fetal CHD among domestic experts of pediatrics in our country, TOP is thought to be an important option if the fetus had a severe CHD, but for some CHDs, especially minor CHDs, the long-term outcomes are optimistic and TOP is not recommended. However, some clinicians are unaware about the need of prenatal genetic tests and counseling, or suggesting termination of pregnancy (TOP) in CHDs with good prognosis, which might lead to unnecessary TOP.

Therefore, we conducted this study to estimate the current level of awareness about providing prenatal genetic tests, explore factors associated with the awareness, attitude towards TOP for fetal CHD and emotion controlling during counseling among clinicians in China.

\section{Methods}

\section{Study Design}

This was a multicenter cross-sectional on-line survey conducted by Xinhua Hospital in October 2017, covering 21 hospital in 7 provinces across China (see supplementary). The study was supported by the Subspecialty Group of Cardiology, Chinese Medical Society of Pediatrics. Ethical approval was obtained from the Institutional Review Board of the Xinhua Hospital affiliated to the Shanghai Jiao Tong University School of Medicine (XHEC-C-2017-070). Informed consents were obtained from all participants.

\section{Participants and Questionnaire}

In total, 501 clinicians were involved in this study, each of whom completed a self-administered questionnaire online, including obstetricians, cardiologists, fetal echocardiography sonographers, and anatomic survey ultrasonographers. Differently from other countries, sonographers, in addition to their specific duties, also play an important role in prenatal genetic service in China, by providing genetic counseling and diagnosis. We divided participants into two groups based on the status of the genetic service in their hospitals. Group 1 included 253 clinicians whose hospitals provided genetic service, which means patients have access to genetic tests and counseling in these hospitals. Group 2 comprised 248 clinicians whose hospitals were not equipped for genetic service. Questionnaire (see supplementary) was designed by three rounds of consultations with experts in prenatal diagnosis, pediatric cardiologists specialized in fetal CHD, and epidemiologists. The validity of the questionnaire and the efficiency of the online system were first tested in a pilot study at the Xinhua Hospital. The questionnaire covered basic demographic information, elementary knowledge of CHD, awareness of prenatal genetic service, cognition on prognosis of fetal CHD, attitude towards TOP and emotional state during prenatal counseling.

We estimated awareness, among clinicians, of prenatal genetic tests, attitudes towards TOP and counseling skills of controlling emotions on the basis of scores of Questions 16, 37 and 39 in questionnaire: (1) Question 16, which kinds of fetal CHD were likely to be associated with specific chromosomal malformations and needed prenatal genetic tests? Respondents had seven choices, i.e., aortic arch anomalies, ventricular septal defect (VSD), tetralogy of Fallot (TOF), transposition of great 
arteries (TGA), pulmonary stenosis (PS), atrial septal defect (ASD), and double outlet of right ventricle (DORV) [1], for five of which the need of genetic service is widely recognized (see supplementary); (2) Question 37, Which kinds of CHD do you think are more possible to have a favorable prognosis and need no suggestion of TOP in most clinical practice? Among 17 choices, five are minor CHDs and are more likely to have favorable outcomes comparing to others, including tricuspid regurgitation (TR), right aortic arch (RAA), VSD, echogenic foci, and mild PS [7]; (3) Question 39, how do you feel if the topic comes to TOP when you are providing prenatal counseling for pregnant women?

In a multiple-choice question, each right choice scored one point and we considered scores of 4 and 5 as high, 2 and 3 as medium, and 0 and 1 as low. A higher score meant a better awareness of providing prenatal genetic tests for patient when necessary and less favor of TOP in minor CHDs. The scores of Question 16 and Question 37 were calculated and statistically analyzed.

\section{Statistical analysis}

All statistical analyses were performed with STATA 14.0 software, and p-values under 0.05 following twotailed $t$ test were considered as statistically significant. A chi-squared test was used to correlate the accuracy of counseling, in the presence of CHD fetuses, with the status of the genetic service.

\section{Results}

\section{Baseline characteristics}

We sent out 982 questionnaires and 549 of them were returned by 21 hospitals with a response rate of $68.8 \%$. A total of 501 clinicians were involved, after excluding 48 clinicians who were not clear of the status of prenatal genetic service in their hospitals, including 108 (21.56\%) obstetricians, 141 (28.14\%) cardiologists, 81 (16.17\%) fetal echocardiography sonographers, and 171 (34.13\%) anatomic survey ultrasonographers at last. Clinicians in group 1 had higher educational levels $(P<0.001)$ and majority of them were working in tertiary hospitals $(P<0.001)$. Over half of clinicians in group 1 were working in Maternal and Child Care Hospitals $(P<0.001)$. Gender $(P=0.998)$ and working years $(P=0.597)$ of clinicians were no difference between two groups (Table 1$)$.

\section{Awareness of providing prenatal genetic tests for fetal CHD}

We found that, regarding the appropriateness of prenatal genetic tests for fetuses with various kinds of fetal CHD, the answers were significantly different between two groups $(P=0.023)$. In total 501 clinicians, only $27.94 \%$ received a high score and $53.09 \%$ received a medium score, indicating a low level of awareness about prenatal genetic tests among clinicians. Clinicians in group 1 obtained higher scores than group 2 (Figure 1). Specifically, 31.62\% clinicians in group 1, but only $24.19 \%$ in group 2 obtained a high score. A low score was obtained by $23.39 \%$ in group 2 compared to $14.62 \%$ in group 1 , reflecting a lower perception of the opportunity to provide prenatal genetic tests for patient in clinicians of group 2 . In addition, groups 1 and 2 did not significantly differ in the proportion of medium scores. The level of awareness was not significantly different between professionals with distinct specialties $(P=0.296)$. 
To control for potential confounders, we performed stepwise multiple linear regression with the level of awareness as the response and all of the other aforementioned variables (Table 1) as predictors. We allowed variable selection to proceed in both directions, namely, forward selection and backward elimination. With the Akaike Information Criterion (AIC) as the target, the best model selected was a regression of the level of awareness on the status of genetic service in hospital, hospital type and the major of clinicians (Table 2). In other words, after taking into account the impact of all variables, the status of genetic service in hospital, hospital type and the major of clinicians were considered to be the most relevant variables to the level of awareness.

\section{Attitude towards TOP for fetal CHD}

We found that attitude towards TOP also varied significantly between these 2 groups $(P=0.023)$. Specifically, a score of 5 was obtained by $44.3 \%$ of clinicians in group 1, but only by $29.4 \%$ of those in group 2 (Figure 2). Among clinicians who achieved a score of 5, who would therefore hold more optimistic attitude and less favor of TOP in minor CHD, group 1 occupied $60.54 \%$. In addition, compared to clinicians who obtained a score of 5 , those who obtained 0 were more likely to suggest TOP in particular CHDs (Figure 3). Independently of the obtained score, completion of pregnancy was always taken into consideration in the case of fetuses with echogenic foci, and over $90 \%$ of clinicians did not suggest TOP.

\section{Counseling skill about controlling emotions during prenatal counseling}

In this question, 309 clinicians indicated that they remained neutral $(P=0.148)$. Of these, 170 clinicians (55.02\%) were from group 1 and 139 (44.98\%) were from group 2 (Table 3). Moreover, 93 clinicians (18.56\%) said they felt sad during prenatal genetic counseling, while others felt anxious $(8.78 \%)$ or depressed (5.19\%). Few of them declared to be confident (1.4\%).

\section{Discussion}

Prenatal diagnosis is important in case of cardiac defects and may improve the outcome of CHDs [8], helping reduce the rate of unnecessary TOP [9]. Prenatal diagnosis can also reduce the genetic burden in the human population [10]. As we know, prenatal genetic tests can effectively assist in prenatal diagnosis, and CHD severity should be evaluated by clinicians through genetic tests. Several different genetic techniques are currently available for prenatal clinical diagnosis, such as chromosome banding for karyotyping [1] and, more recently, fluorescent in situ hybridization (FISH). Meanwhile, array comparative genomic hybridization (aCGH) [11] and non-invasive prenatal testing [12] are alternative options. However, education and expertise are still inadequate for optimal CHD recognition and management. Our study showed that many clinicians exhibited insufficient awareness regarding the provision of genetic services to patients. In China, the implementation of clinical genetic services is scarce [4]. There are still lots of hospitals have no access to prenatal genetic service for patients and clinicians lack of elementary knowledge of prenatal management of $\mathrm{CHD}$. Establishment of prenatal genetic service system and development of educational programs on genetics by the healthcare system are warranted and desired [13]. 
Prenatal genetic counseling implies accurate diagnosis of CHD and clear explanation of complex medical issues to parents, including management options, long term functional and neurodevelopmental for fetuses outcomes. Our study indicated that whether had access to prenatal genetic services in hospitals influenced the judgement by clinicians on prognosis of fetal CHD. It is also important that counselors have good communication skills and show empathy to parents [14]. Parental perception is heavily affected by the manner in which this information is conveyed, and considerable variability remains in the quality of counseling [15]. Emotional control is another important issue that may seriously affect the validity of counseling. At present, there are no specialty-trained geneticists or genetic counselors in China, and pediatricians, obstetricians, as well as sonographers (the latter also taking part in prenatal diagnosis), may be in charge of providing genetic counseling to patients. Therefore, a nationwide education platform focused on prenatal genetic counseling is urgently needed.

Once fetal CHD has been diagnosed, TOP is one of the possible options if necessary. It was reported that the higher the number of prenatal tests, the higher the percentage of TOP [16]. Moreover, Chinese women were found to be more prone to accept the possibility of abortion in the event of a fetal abnormality, compared to English women [17]. In some cases, couples would decide to terminate a pregnancy to avoid putting families to shame [18]. A previous study showed that shame and stigma of disability accompanied by social and family pressure [19] may influence parental decisions, especially in Asia. An incorrect judgement by clinicians on prognosis of fetal CHD during counseling could not help parents in understanding the progress and prognosis of the disease and may lead to unnecessary TOP, especially in the presence of minor CHD. Thus, training on counseling techniques and improving the cognition of fetal CHDs among clinicians are important to reduce the rate of unnecessary TOP in China.

Clinical genetic services are poorly utilized and should be promoted in most hospitals in China. Training and education of obstetricians, cardiologists, and fetal echocardiography sonographers is vital to improve the genetic services in hospitals. In cases where, due to complex situations related to pregnancy management and outcome, clinicians are not able to provide appropriate counseling for fetal CHD, they should have the option to refer to a Fetal Medicine Unit, where accurate genetic testing and counseling can be performed. Fetuses with a prenatal diagnosis of major CHD should be delivered in tertiary referral centers [20] where clinical management and prognosis can be provided by multidisciplinary teams, after discussion of all relevant aspects involved [21].

Also, larger and more comprehensive studies are needed for an in-depth evaluation of the current status of prenatal genetic services in China, also covering clinician education and hospital management.

Since this survey mostly involved tertiary hospitals, the major limitation of the study concerns the generalizability of the results to the whole health-care system in China.

\section{Conclusion}

In this study, we found that a remarkable proportion of clinicians showed poor awareness of the need to provide genetic service for fetal CHD, especially those working in hospitals that did not offer prenatal 
genetic services. Moreover, the attitudes towards TOP of the latter group was less optimistic as well. Thus, both the awareness of prenatal genetic service and the cognition on prognosis of fetal CHD should be improved through reformation of establishing genetic service in China.

\section{Declarations}

Ethics approval and consent to participate: Ethic approval was obtained from the Institutional Review Broad of Xinhua Hospital affiliated to the Shanghai Jiao Tong University School of Medicine. Informed consents were obtained from all participants.

Consent for publication: Consent for publication was obtained from each patient.

Availability of data and materials: The datasets used and/or analyzed during the current study are available from the corresponding author on reasonable request.

Competing interests: The authors declare that they have no competing interests.

Funding: 1. Shanghai municipal health commission, 20184Y0062 2. Ministry of Science and Technology of the People's Republic of China, 2018YFC1002400

Author contribution: Jian Wang designed this study and analyzed the data received from each hospital; Zhuoyan Li drafted the article and confirm the validity of data; Jianping Yang, Yanan Lu and Yurong Wu revised the article and gave technical advice of the whole research; Ailan Xie, Yongwei Zhang, Jiannong Chai, Bo Han, Yuqing Zhou and Tiangang Li acquired the data of their working hospital and took the responsibility of implementation of the research in sub-center; Sun Chen and Kun Sun are corresponding authors of this article and they took responsibility of the whole research.

Acknowledgements: We thank all the clinicians for participating in the interviews.

\section{References}

1. Donofrio MT, Moon-Grady AJ, Hornberger LK, Copel J A, Sklansky MS, Abuhamad A, et al. Diagnosis and treatment of fetal cardiac disease: a scientific statement from the American Heart Association. Circulation 2014; 129: 2183-2242.

2. Song MS, Hu A, Dyamenahalli U, Chitayat D, Winsor EJ, Ryan G, et al. Extracardiac lesions and chromosomal abnormalities associated with major fetal heart defects: comparison of intrauterine, postnatal and postmortem diagnoses. Ultrasound Obstet Gynecol 2009; 33: 552-559.

3. Lytzen R, Vejlstrup N, Bjerre J, Petersen OB, Leenskjold S, Dodd JK, et al. Live-born major congenital heart disease in Denmark: incidence, detection rate, and termination of pregnancy rate from 1996 to 2013. JAMA Cardiol 2018; 3: 829-837.

4. Zhang D, Ng VH, Wang Z, Zhai X, Lie RK. Eugenics and mandatory informed prenatal genetic testing: a unique perspective from China. Dev World Bioeth 2016; 16: 107-115. 
5. Abacan M, Alsubaie L, Barlow-Stewart K, Caanen B, Cordier C, Courtney E, et al. The global state of the genetic counseling profession. Eur J Hum Genet 2019; 27: 183-197.

6. Killen SA, Mouledoux JH, Kavanaugh-McHugh A. Pediatric prenatal diagnosis of congenital heart disease. Curr Opin Pediatr 2014; 26: 536-545.

7. Sun $\mathrm{K}$, Chen SB, Han I. Expert consensus on diagnosis and perinatal management of fetal congenital heart disease. Chinese Journal of Pediatrics 2015; 53: 728-733.

8. Bravo-Valenzuela NJ, Peixoto AB, Araujo Junior E. Prenatal diagnosis of congenital heart disease: a review of current knowledge. Indian Heart J 2018; 70: 150-164.

9. Guerra B, Simonazzi G, Banfi A, Lazzarotto T, Farina A, Lanari M, et al. Impact of diagnostic and confirmatory tests and prenatal counseling on the rate of pregnancy termination among women with positive cytomegalovirus immunoglobulin M antibody titers. Am J Obstet Gynecol 2007; 196: 221 e221-e226.

10. Verma IC, Puri RD. Global burden of genetic disease and the role of genetic screening. Semin Fetal Neonatal Med 2015; 20: 354-363.

11. Jansen FA, Blumenfeld YJ, Fisher A, Cobben JM, Odibo AO, Borrell A, et al. Array comparative genomic hybridization and fetal congenital heart defects: a systematic review and meta-analysis. Ultrasound Obstet Gynecol 2015; 45: 27-35.

12. Jensen TJ, Dzakula Z, Deciu C, van den Boom D, Ehrich M. Detection of microdeletion 22q112 in a fetus by next-generation sequencing of maternal plasma. Clin Chem 2012; 58: 1148-1151.

13. De Groote K, Vanhie E, Roets E, Ramaekers P, De Wilde H, Panzer J, et al. Outcome after prenatal and postnatal diagnosis of complex congenital heart defects and the influence of genetic anomalies. Prenat Diagn 2017; 37: 983-991.

14. Lee CK. Prenatal Counseling of Fetal Congenital Heart Disease. Curr Treat Options Cardiovasc Med 2017; 19: 5.

15. Hunter LE, Simpson JM. Prenatal screening for structural congenital heart disease. Nat Rev Cardiol 2014; 11: 323-334.

16. A Gaboon NE, Bakur KH, Edrees AY, Al-Aama JY. Attitude toward prenatal testing and termination of pregnancy among health professionals and medical students in Saudi Arabia. J Pediatr Genet 2017; 6: $149-154$.

17. Li G, Chandrasekharan S, Allyse M. "The top priority is a healthy baby": narratives of health, disability, and abortion in online pregnancy forum discussions in the US and China. J Genet Couns 2017; 26: 32-39.

18. Chiu M Y, Yang X, Wong FH, Li JH, Li J. Caregiving of children with intellectual disabilities in China-an examination of affiliate stigma and the cultural thesis. J Intellect Disabil Res 2013; 57: 1117-1129.

19. Tsai GJ, Cameron CA, Czerwinski JL, Mendez-Figueroa H, Peterson SK, Noblin SJ. Attitudes towards prenatal genetic counseling, prenatal genetic testing, and termination of pregnancy among Southeast and East Asian women in the United States. J Genet Couns 2017; 26: 1041-1058. 
20. Paladini D, Alfirevic Z, Carvalho JS, Khalil A, Malinger G, Martinez JM, et al. ISUOG consensus statement on current understanding of the association of neurodevelopmental delay and congenital heart disease: impact on prenatal counseling. Ultrasound Obstet Gyneco/ 2017; 49: 287-288.

21. Wimalasundera RC, Gardiner HM. Congenital heart disease and aneuploidy. Prenat Diagn 2004; 24: 1116-1122.

\section{Tables}

Table 1. Basic characteristics of 501 clinicians.

\begin{tabular}{|c|c|c|c|}
\hline & Genetic(group 1) & No genetic(group 2) & P-Value \\
\hline Total & 253 & 248 & \\
\hline Gender & & & 0.998 \\
\hline Male & $52(20.55)$ & $51(20.56)$ & \\
\hline Female & $201(79.45)$ & $197(79.44)$ & \\
\hline Clinician Catalogs & & & 0.011 \\
\hline Obstetrician & $59(23.32)$ & $49(19.76)$ & \\
\hline Cardiologist & $84(33.20)$ & $57(22.98)$ & \\
\hline Fetal Echocardiography Sonographer & $39(15.42)$ & $42(16.94)$ & \\
\hline Anatomic Survey Ultrasonographer & $71(28.06)$ & $100(40.32)$ & \\
\hline Educational Level & & & $<0.001$ \\
\hline Doctor & $27(10.67)$ & $7(2.82)$ & \\
\hline Post-graduate & $100(39.53)$ & $45(18.15)$ & \\
\hline Undergraduate & $120(47.43)$ & $175(70.56)$ & \\
\hline Junior College \& Polytechnic School & $6(2.38)$ & $21(8.46)$ & \\
\hline Working Years & & & 0.597 \\
\hline $0-4$ & $62(24.51)$ & $57(22.98)$ & \\
\hline $5-9$ years & $70(27.67)$ & $81(32.66)$ & \\
\hline 10-14 years & $50(19.76)$ & $50(20.16)$ & \\
\hline$\geq 15$ years & $71(28.06)$ & $60(24.19)$ & \\
\hline Professional Title & & & 0.006 \\
\hline Chief physician & $30(11.86)$ & $17(6.85)$ & \\
\hline Associate chief physician & $46(18.18)$ & $55(22.18)$ & \\
\hline Physician & $101(39.92)$ & $126(50.81)$ & \\
\hline Resident & $75(29.64)$ & $47(18.95)$ & \\
\hline Hospital Level & & & $<0.001$ \\
\hline Tertiary & $240(94.86)$ & $192(77.42)$ & \\
\hline Secondary & $13(5.14)$ & $53(21.37)$ & \\
\hline Hospital Type & & & $<0.001$ \\
\hline General Hospitals & $78(30.83)$ & $137(55.24)$ & \\
\hline Children's Hospitals & $33(13.04)$ & $18(7.26)$ & \\
\hline Maternal and Child Care Hospitals & $137(54.15)$ & $80(32.26)$ & \\
\hline Private Hospitals & $5(1.98)$ & $13(5.24)$ & \\
\hline
\end{tabular}


Table 2. The stepwise multiple linear regression analysis of the level of awareness.

\begin{tabular}{lcccc} 
& Coef. & Std. Error & $\mathrm{t}$-value & $\mathrm{P}(>|\mathrm{t}|)$ \\
\hline Genetic in Hospital & .1405227 & .0452311 & 3.11 & 0.002 \\
\hline Hospital Type & .0663285 & .02252 & 2.95 & 0.003 \\
Major & -.0216929 & .0086234 & -2.52 & 0.012
\end{tabular}

Table 3. Comparisons of clinicians' emotions during prenatal counseling for pregnancies with fetal CHD in two groups.

\begin{tabular}{lcccccccc}
\hline & \multicolumn{7}{c}{$\begin{array}{l}\text { Emotion } \\
(\mathrm{n}, \%)\end{array}$} \\
\cline { 2 - 8 } & Anxiety & Sadness & Confidence & Neutrality & Depression & Other & Total & $\begin{array}{r}\text { P- } \\
\text { value }\end{array}$ \\
\hline All & $44(8.78)$ & $93(18.56)$ & $7(1.4)$ & $309(61.68)$ & $26(5.19)$ & $22(4.39)$ & 501 & 0.148 \\
$\begin{array}{l}\text { Genetic } \\
\text { in } \\
\text { hospital }\end{array}$ & & & & & & & & \\
$\quad$ Yes & $16(36.36)$ & $42(45.16)$ & $4(57.14)$ & $170(55.02)$ & $11(42.31)$ & $10(45.45)$ & $253(50.5)$ & \\
\multicolumn{1}{c}{ No } & $28(63.64)$ & $51(54.84)$ & $3(42.86)$ & $139(44.98)$ & $15(57.69)$ & $12(55.55)$ & $248(49.5)$ &
\end{tabular}

Figures 


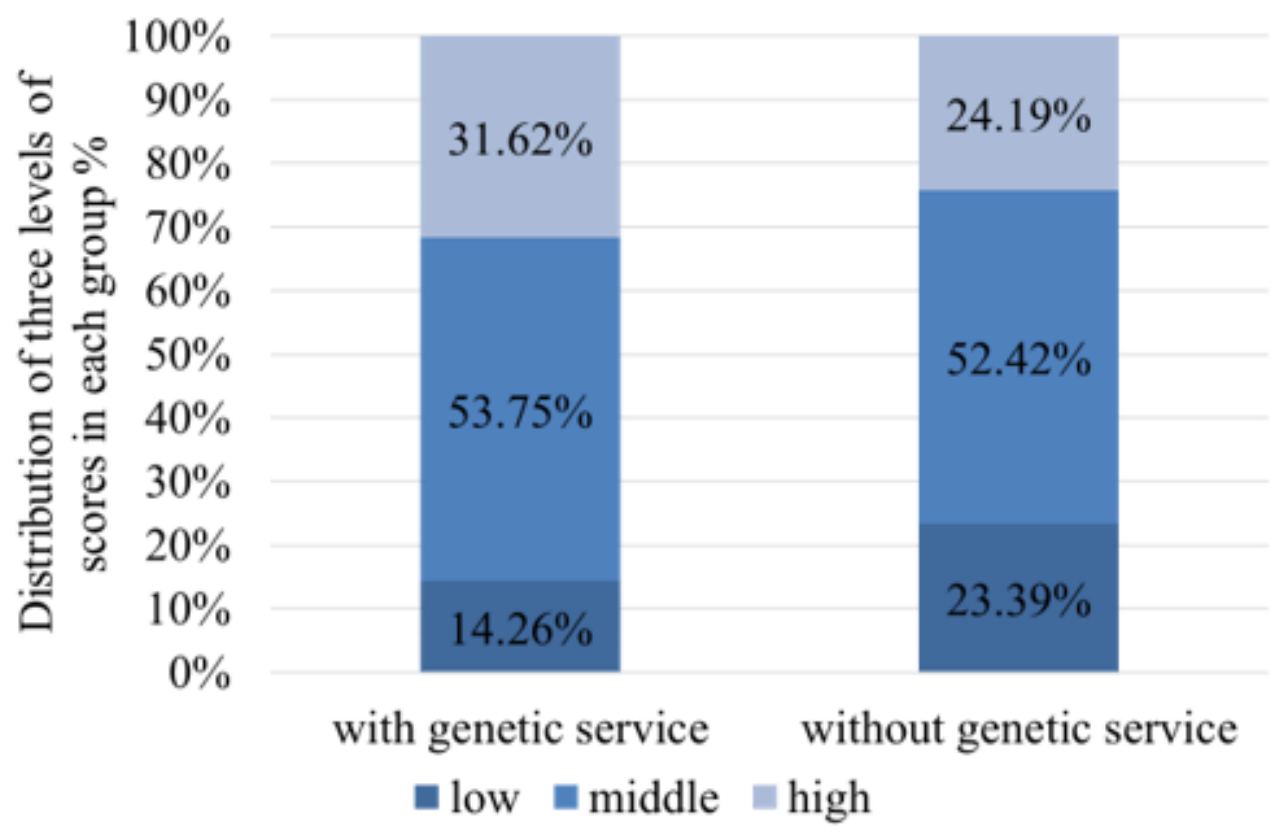

Figure 1

On Question 16, 31.62\% clinicians in group 1 obtained a high score, while $24.19 \%$ in group 2 got the same scores. Group 1 had higher perceptions of the opportunity to provide prenatal genetic tests for fetal CHD.

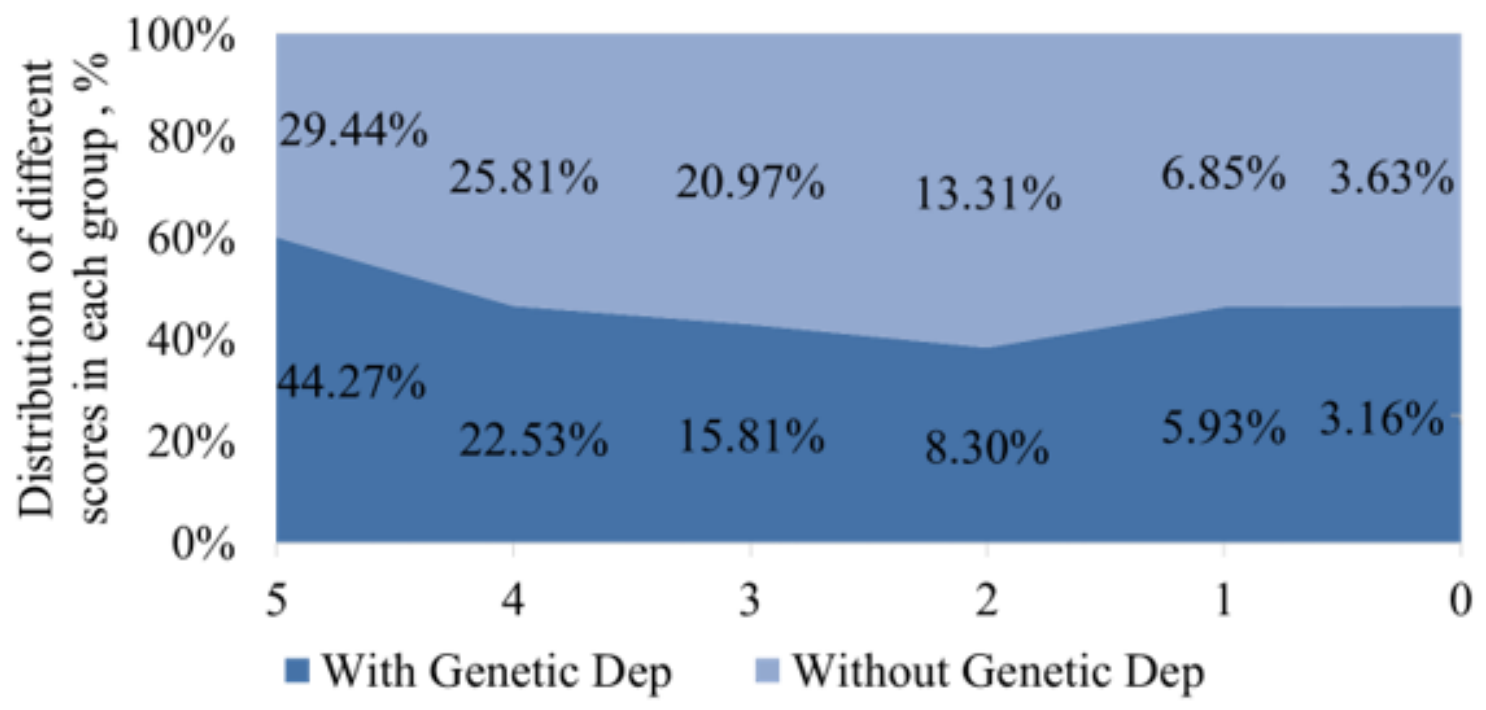

\section{Figure 2}

The distribution of different scores in two groups on Question 37. About 44.3\% of group 1 obtained a score of 5 but only $29.4 \%$ of group 2 got the same score. Clinicians whose working hospitals have access 
to genetic service are more possible to give more appropriate advices based on the prognosis of fetal CHD during counseling.

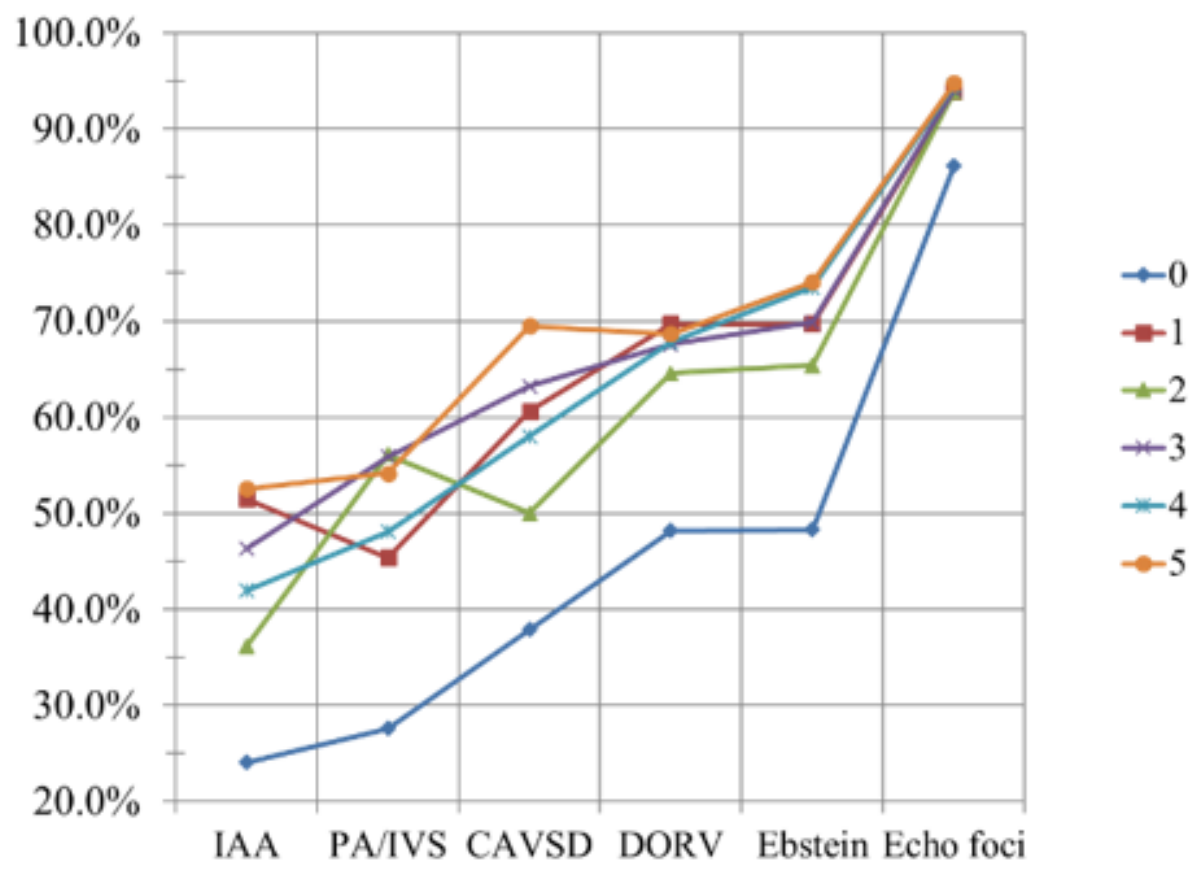

\section{Figure 3}

The percentage of suggesting no TOP in particular CHDs by different scores. Clinicians who achieved a score of 0 were more favor of TOP than those who obtained a high score. No matter what scores they got, continuing pregnancy was always taken into consider when they confronted fetuses with echogenic foci and over $90 \%$ of clinicians suggest no TOP.

\section{Supplementary Files}

This is a list of supplementary files associated with this preprint. Click to download.

- supplementary.doc 